International Mathematical Forum, Vol. 8, 2013, no. 39, 1921 - 1931

HIKARI Ltd, www.m-hikari.com

http://dx.doi.org/10.12988/imf.2013.310194

\title{
Adaptive Finite Elements with Thin Obstacles
}

\author{
J. Frohne and F.T. Suttmeier \\ Department Mathematik \\ Universität Siegen \\ D-57258 Siegen, Germany
}

Copyright (c) 2013 J. Frohne and F.T. Suttmeier. This is an open access article distributed under the Creative Commons Attribution License, which permits unrestricted use, distribution, and reproduction in any medium, provided the original work is properly cited.

\begin{abstract}
This work describes concepts for a posteriori error estimation and adaptive mesh design for dual-mixed finite element models where the solution is subjected to inequality constraints. These methods are developed here especially for variational problems with a so-called thin obstacle, i.e. the restrictions are imposed on the boundary of the domain under consideration. The studies presented in this work are partly motivated by our memberships in research groups, where one focus is on the numerical FE-analysis of 3D-contact problems. Eventually, numerical tests confirm our theoretical results.
\end{abstract}

Keywords: obstacle problem, a posteriori error estimate, variational inequality, finite element method, adaptivity

\section{Introduction}

The studies presented in this work are partly motivated by our memberships in some research groups supported by the Deutsche Forschungsgemeinschaft (DFG). In collaboration with scientists from mechanical engineering, we provide the numerical analysis for problems arising in the field of continuum mechanics.

In this context (c.f. Suttmeier [15]), systems of partial differential equations, where the solution is subjected to inequality constraints, have to be treated. We employ the finite element Galerkin (FE) method to obtain approximate solutions of such systems, which for instance typically arise in the 
field of contact mechanics. Examples are plastic materials, where certain norms of the stresses are bounded or unilateral problems, where the displacement is restricted by a rigid obstacle. The basis for applying an FE discretisation is a suitable mathematical setting, which in the topics under consideration takes the form of variational inequalities (VI).

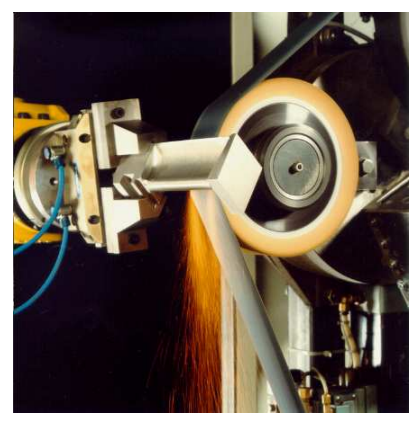

Figure 1: Snap shot of a grinding process.

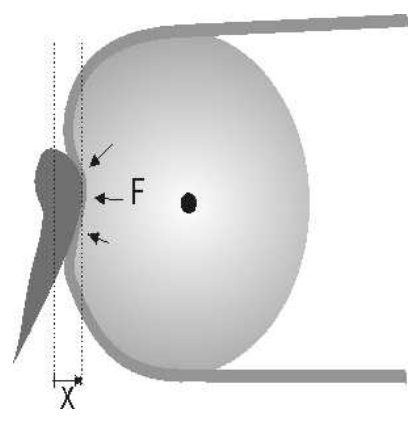

Figure 2: Sketch of the model situation.

For illustration, the situation of a workpiece pressed onto a grinding disk (Figures 1,2) is approximated by a FE-scheme (Figures 3,4) to approximate the resulting surface forces.

The underlying, mathematical model situation for contact problems in elasticity is Signorini's problem, which in classical notation takes the form (cf. Kikuchi and Oden [8])

$$
\begin{gathered}
-\operatorname{div} \sigma=f, \quad A \sigma=\varepsilon(u) \quad \text { in } \Omega, \\
\left.\begin{array}{c}
u=0 \text { on } \Gamma_{D}, \quad \sigma \cdot n=t \\
\sigma_{T}=0, \quad\left(u_{n}-G\right) \sigma_{n}=0 \\
u_{n}-G \leq 0, \quad \sigma_{n} \leq 0
\end{array}\right\} \text { on } \Gamma_{C} .
\end{gathered}
$$

This idealised model describes the deformation of an elastic body occupying the domain $\Omega \subset \mathbb{R}^{3}$, which is unilaterally supported by a frictionless rigid foundation. The boundary $\partial \Omega$ is assumed to be divided into non-empty subsets $\Gamma_{N}, \Gamma_{D}, \Gamma_{C}$, which have no points in common. The displacement $u$ and the corresponding stress tensor $\sigma$ are caused by a body force $f$ and a surface traction $t$ along $\Gamma_{N}$. Along the portion $\Gamma_{D}$ of the boundary the body is fixed and $\Gamma_{C} \subset \partial \Omega$ denotes the part which is a candidate contact surface. We use the notation $u_{n}=u \cdot n, \sigma_{n}=\sigma_{i j} n_{i} n_{j}$ and $\sigma_{T}=\sigma \cdot n-\sigma_{n} n$, where $n$ is the outward normal of $\partial \Omega$, and $G$ denotes the gap between $\Gamma_{C}$ and the foundation.

Further, the deformation is supposed to be small, so that the strain tensor can be written as $\varepsilon(u)=\frac{1}{2}\left(\nabla u+\nabla u^{T}\right)$. The compliance tensor $A$ is assumed 
to be symmetric and positive definite. We intend to derive a posteriori error control techniques for this equation.

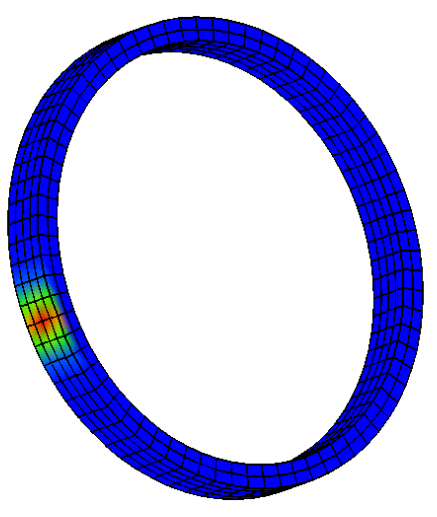

Figure 3: FE-Simulation

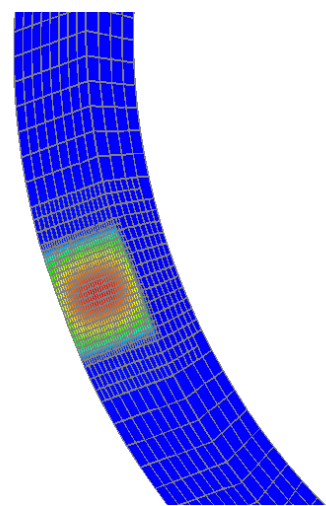

Figure 4: Zoom.

In order to demonstrate the concept for our method for a posteriori error estimation, we consider the simplified case

$$
\begin{gathered}
-\Delta u=f \quad \text { in } \Omega \subset \mathbb{R}^{2}, \\
u=0 \quad \text { on } \Gamma_{D} \neq \emptyset, \\
u-g \geq 0, \quad \partial_{n} u \geq 0, \quad(u-g) \partial_{n} u=0 \quad \text { on } \Gamma_{C},
\end{gathered}
$$

where $\Gamma_{C}=\partial \Omega \backslash \Gamma_{D}$ and $\partial_{n} u=\nabla u \cdot n . g$ is a given function denoting the obstacle along the boundary.

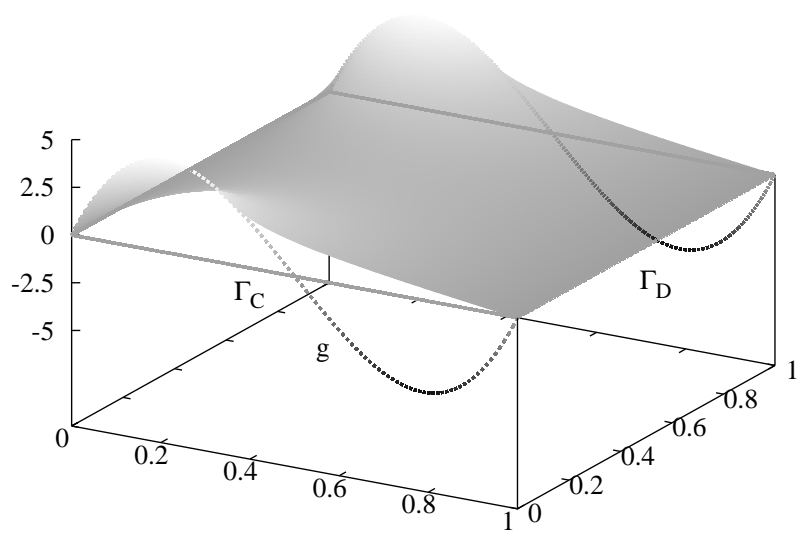

Figure 5: Illustration of obstacle condition along $\Gamma_{C} \subset \partial \Omega$. 
Problem (2) is to be solved by the finite element Galerkin method on adaptively optimized meshes.

The basis for applying the finite element method to (2) is the formulation as a variational inequality. Here as first option a solution $u \in K$ is sought fulfilling

$$
(\nabla u, \nabla(\varphi-u)) \geq(f, \varphi-u) \quad \forall \varphi \in K
$$

where we set $V=\left\{v \in H^{1} \mid v=0\right.$ on $\left.\Gamma_{D}\right\}$ and $K=\left\{v \in V \mid v \geq g\right.$ on $\left.\Gamma_{C}\right\}$.

Here and in what follows, (., .) represents the $L^{2}$ inner product of a bounded domain $\Omega$ in $\mathbb{R}^{2}$ and $\|$.$\| the corresponding norm. Furthermore H^{m}=H^{m}(\Omega)$ denotes the standard Sobolev space of $L^{2}$-functions with derivatives in $L^{2}(\Omega)$ up to the order $m$, and $H_{0}^{1} \subset H^{1}$ is the subspace of $H^{1}$-functions vanishing on $\partial \Omega$. Existence of a unique solution can be established by using the theoretical framework presented e.g. in Glowinski [7].

We will apply the finite element method on decompositions $\mathbb{T}_{h}=\left\{T_{i} \mid 1 \leq\right.$ $\left.i \leq N_{h}\right\}$ of $\Omega$ consisting of $N_{h}$ triangular elements $T_{i}$, satisfying the usual condition of shape regularity. For ease of mesh refinement and coarsening, hanging nodes are allowed in our implementation. The width of the mesh $\mathbb{T}_{h}$ is characterised in terms of a piecewise constant mesh size function $h=h(x)>0$, where $h_{T}:=h_{\mid T}=\operatorname{diam}(T)$. Eventually, following Blum \& Suttmeier [2], the finite element approximation $u_{h}$ of $u$ in (3) is determined by

$$
\left(\nabla u_{h}, \nabla\left(\varphi-u_{h}\right)\right) \geq\left(f, \varphi-u_{h}\right) \quad \forall \varphi \in K_{h},
$$

with $K_{h}=V_{h} \cap K$, where $V_{h}$ by standard linear finite elements and for simplicity $g$ is assumed to be polygonal. This finite dimensional problem can be shown to be uniquely solvable following the same line of arguments as in the continuous case. Optimal order a priori error estimates in the energy norm have been given for example in Falk [6] and Brezzi et al. [3]. Dobrowolski and Staib [5] show $\mathcal{O}(h)$-convergence in the energy norm without additional assumptions on the structure of the free boundary. Error estimates with respect to the $L^{\infty}$-norm have been obtained, e.g., by Nitsche [9] based on a discrete maximum principle.

\section{$2 \quad$ A mixed setting}

In general (c.f. Suttmeier [14]), for applying a finite element method to a given problem, it has to be written in a variational setting, which is accessible for a Galerkin scheme. Additional constraints, as for example the incompressibility condition in the Stokes problem, have to be considered as restrictions. These can be treated by the Lagrangian formalism yielding saddle point problems. 
One important application of such mixed systems and the corresponding finite element schemes is the following: For higher order problems one defines auxiliary variables for the derivatives. The equations describing these new quantities are handled as restrictions in the reformulated problem. In this way certain derivatives represented by auxiliary variables are approximated with higher accuracy. Additionally the new formulation often allows to weaken the regularity assumptions on the primary solution. As one example we mention problems in perfect plasticity. Here, discontinuities may occur in the displacement field $u$ due to slip lines in the micro-structure, whereas the stresses $\sigma$, determined by linear combinations of derivatives of $u$, have a smoother behaviour (see Suquet [12], Seregin [11]). From a practical point of view, the stresses are required with high accuracy. Therefore mixed methods treating the stresses directly are often more adequate for solving problems in continuum mechanics.

In our case, introducing the stress vector $\sigma=\left(\sigma_{1}, \sigma_{2}\right)$, the problem can be stated in form of a first order system,

$$
\begin{gathered}
\sigma=\nabla u, \\
-\operatorname{div} \sigma=f, \\
u=0 \quad \text { on } \Gamma_{D}, \\
u-g \geq 0, \quad \sigma n \geq 0, \quad(u-g) \cdot \sigma n=0 \quad \text { on } \Gamma_{C} .
\end{gathered}
$$

The dual-mixed variational formulation of (5) reads, see e.g. Wang \& Yang [16],

$$
\begin{gathered}
(\sigma, \tau-\sigma)+(u, \operatorname{div} \tau-\operatorname{div} \sigma) \geq \int_{\Gamma_{C}} g(\tau-\sigma) n d \Gamma \quad \forall \tau \in H \\
-(\operatorname{div} \sigma, \varphi)=(f, \varphi) \quad \forall \varphi \in L^{2}
\end{gathered}
$$

with $H:=\left\{\tau \in H_{\text {div }}(\Omega) \mid \tau \cdot n \geq 0\right\}$ and $H_{\text {div }}(\Omega)=\left\{\tau \in L^{2}(\Omega)^{2}, \operatorname{div} \tau \in L^{2}(\Omega)\right\}$.

Exploiting $-\operatorname{div} \sigma=f$ so called least-squares concepts may be applied to obtain

$$
\begin{gathered}
a(\sigma, \tau-\sigma)+(u, \operatorname{div}(\tau-\sigma)) \geq-\delta(f, \operatorname{div}(\tau-\sigma))+\int_{\Gamma} g(\tau-\sigma) n d \Gamma \quad \forall \tau \in H \\
-(\operatorname{div} \sigma, \varphi)=(f, \varphi) \quad \forall \varphi \in L^{2}
\end{gathered}
$$

with $a(.,):.=(.,)+.\delta(\operatorname{div} ., \operatorname{div}$.$) and suitable \delta \geq 0$.

The discrete analogue seeks for a pair $\left\{\sigma_{h}, u_{h}\right\}$ fulfilling

$$
\begin{aligned}
& a\left(\sigma_{h}, \tau-\sigma_{h}\right)+\left(u_{h}, \operatorname{div}\left(\tau-\sigma_{h}\right)\right)-\left(\operatorname{div} \sigma_{h}, \varphi\right)+\delta_{h}\left(\left[u_{h}\right],[\varphi]\right) \geq \\
& \quad-\delta\left(f, \operatorname{div}\left(\tau-\sigma_{h}\right)\right)+\int_{\Gamma} g\left(\tau-\sigma_{h}\right) n d \Gamma+(f, \varphi), \forall\{\tau, \varphi\} \in H_{h} \times W_{h}
\end{aligned}
$$


The finite dimensional space $H_{h} \times W_{h} \subset H \times L^{2}$ for a discretisation for this saddle point problem is determined by approximating each component of the stresses by the standard linear shape functions for $H_{h}$. $W_{h}$ is constructed by elementwise constant functions. Above the brackets [.] denote the jump across the element boundaries $\partial T$ and $\delta_{h}$ is an appropriatly chosen mesh-dependent parameter. For details to this notation we refer to Becker [1]. In computations below we choose $\delta_{h}=\mathcal{O}(h)$

\section{A posteriori error analysis}

In what follows, we present the error analysis for the discretisation error $\left(e, e^{p}\right)=\left(u-u_{h}, p-p_{h}\right)$ between the continuous problem (7) and the discrete system (8). Below, the subscript $i$ denotes the standard interpolant of its argument.

Additionally defining $\|\tau\|_{a}^{2}:=\|\tau\|^{2}+\delta\|\operatorname{div} \tau\|^{2}$ one obtains

$$
\begin{array}{r}
\left\|\sigma-\sigma_{h}\right\|_{a}^{2}=\left(\sigma-\sigma_{h}, \sigma-\sigma_{h}\right)+\left(u-u_{h}, \operatorname{div}\left(\sigma-\sigma_{h}\right)\right)+\delta\left\|f+\operatorname{div} \sigma_{h}\right\|^{2} \\
-\left(\operatorname{div}\left(\sigma-\sigma_{h}\right), u-u_{i}\right)-\left(\operatorname{div}\left(\sigma-\sigma_{h}\right), u_{i}-u_{h}\right)
\end{array}
$$

Here the last term may be controlled as follows. We start with taking the difference between $(7)$ and (8) tested with pairs $\left(0, u_{i}-u_{h}\right)$, yielding

$$
\left(-\operatorname{div}\left(\sigma-\sigma_{h}\right), u_{i}-u_{h}\right)=\delta_{h}\left(\left[u_{h}\right],\left[u_{i}-u_{h}\right]\right) .
$$

Now locally, using the standard trace theorem, one proceeds by

$$
\begin{aligned}
\delta_{h}\left(\left[u_{h}\right],\left[u_{i}\right]\right)_{T} & \leq \delta_{h}\left(\left[u_{h}\right], u_{i}^{l}-u\right)_{\partial T}+\delta_{h}\left(\left[u_{h}\right], u-u_{i}^{r}\right)_{\partial T} \\
& \leq c \delta_{h}\left(\left[u_{h}\right],\left[u_{h}\right]\right)_{\partial T}+\delta_{h} h_{T}\|\nabla u\|_{T}^{2} \\
& \leq c \delta_{h}\left(\left[u_{h}\right],\left[u_{h}\right]\right)_{\partial T}+\delta_{h} h_{T}\left\|\sigma_{h}\right\|_{T}^{2}+\delta_{h} h_{T}\left\|\sigma-\sigma_{h}\right\|_{T}^{2}
\end{aligned}
$$

where $u_{i}^{l}$ and $u_{i}^{r}$ denote the values of $u_{i}$ on the two triangles having an edge of $\partial T$ in common. Furthermore we set

$$
J_{h}:=\sum_{T} c \delta_{h}\left(\left[u_{h}\right],\left[u_{h}\right]\right)_{\partial T}+\delta_{h} h_{T}\left\|\sigma_{h}\right\|_{T}^{2}+\delta_{h} h_{T}\left\|\sigma-\sigma_{h}\right\|_{T}^{2} .
$$

Next, introducing $u_{L} \in V_{h}$ as a suitable approximation of $u_{h}$ with the additional property $u_{L}-g \geq 0$ on $\Gamma_{C}$, we get

$$
\begin{aligned}
\left\|\sigma-\sigma_{h}\right\|_{a}^{2} \leq J_{h}+\left(-\sigma_{h}, \sigma-\sigma_{h}\right)-\left(u_{L}, \operatorname{div}\left(\sigma-\sigma_{h}\right)\right)+\int_{\Gamma} g\left(\sigma-\sigma_{h}\right) n d \Gamma \\
+\left(u_{L}-u_{h}, \operatorname{div}\left(\sigma-\sigma_{h}\right)\right)+\delta\left\|f+\operatorname{div} \sigma_{h}\right\|^{2}-\left(\operatorname{div}\left(\sigma-\sigma_{h}\right), u-u_{i}\right) .
\end{aligned}
$$


Remark: Since $u_{h}$ is a cell-wise constant function, $u_{L}$ may easily be obtained by local averaging techniques (Zienkiewicz \& Zhu [17]) and a simple postprocess ensuring $u_{L}-g \geq 0$ on $\Gamma_{C}$.

Recalling the inequality $\sigma n \geq 0$ one obtains

$$
\begin{aligned}
&\left\|\sigma-\sigma_{h}\right\|_{a}^{2} \leq J_{h}+\left(\nabla u_{L}-\sigma_{h}, \sigma-\sigma_{h}\right)-\int_{\Gamma}\left(u_{L}-g\right) \cdot\left(\sigma-\sigma_{h}\right) n d \Gamma \\
&+\delta\left\|f+\operatorname{div} \sigma_{h}\right\|^{2}+\left(u_{L}-u_{h},-f-\operatorname{div} \sigma_{h}\right)+\left(\operatorname{div} \sigma_{h}+f, u-u_{i}\right) \\
& \leq J_{h}+\left(\nabla u_{L}-\sigma_{h}, \sigma-\sigma_{h}\right)+\int_{\Gamma}\left(u_{L}-g\right) \cdot \sigma_{h} n d \Gamma+\delta\left\|f+\operatorname{div} \sigma_{h}\right\|^{2} \\
&+\left(u_{h}-u_{L}+u-u_{i}, f+\operatorname{div} \sigma_{h}\right) .
\end{aligned}
$$

Using interpolation gives us

$$
\begin{aligned}
c\left\|\sigma-\sigma_{h}\right\|_{a}^{2} \leq \| \nabla u_{L} & -\sigma_{h}\left\|^{2}+\int_{\Gamma}\left(u_{L}-g\right) \cdot \sigma_{h} n d \Gamma+\delta\right\| f+\operatorname{div} \sigma_{h} \|^{2} \\
& +\sum_{T}\left(\left\|u_{L}-u_{h}\right\|_{T}+c_{i} h_{T}\|\nabla u\|_{T}\right)\left\|f+\operatorname{div} \sigma_{h}\right\|_{T}+J_{h},
\end{aligned}
$$

with a constant $c>0$. Eventually $\nabla u=\sigma-\sigma_{h}+\sigma_{h}$ gives

$$
c\left\|\sigma-\sigma_{h}\right\|_{a}^{2} \leq \eta\left(\sigma_{h}, u_{h}, f, g\right)
$$

with the definition

$$
\begin{aligned}
\eta\left(\sigma_{h}, u_{h}, f, g\right) & :=\sum_{T}\left(\left\|\nabla u_{L}-\sigma_{h}\right\|_{T}^{2}+\left\|u_{L}-u_{h}\right\|_{T}^{2}+h_{T}^{2}\left\|\sigma_{h}\right\|_{T}^{2}\right) \\
& +\sum_{T} c \delta_{h}\left(\left[u_{h}\right],\left[u_{h}\right]\right)_{\partial T}+\sum_{T}\left\|f+\operatorname{div} \sigma_{h}\right\|_{T}^{2}+\int_{\Gamma}\left(u_{L}-g\right) \cdot \sigma_{h} n d \Gamma .
\end{aligned}
$$

Consequently, observing $\left\|\sigma-\sigma_{h}\right\|_{\text {div }}=\left\|\sigma-\sigma_{h}\right\|+\left\|f+\operatorname{div} \sigma_{h}\right\|$, we can state

Theorem 3.1. For the discretisation error $\sigma-\sigma_{h}$ measured in the natural $H_{\mathrm{div}}$-norm there holds the "a posteriori estimate"

$$
\left\|\sigma-\sigma_{h}\right\|_{\text {div }}^{2} \leq c \eta\left(\sigma_{h}, u_{h}, f, g\right),
$$

with notations introduced above.

\section{Numerical test}

The numerical results presented throughout this work are obtained by FE-implementations based on the DEAL-library $[4,13]$. 
The a posteriori mesh design is organised as follows. Let an error tolerance TOL or a maximal number of cells $N_{\max }$ be given. Starting from some initial coarse mesh the refinement criteria are chosen in terms of the local error indicators $\eta_{T}$. Then for the mesh refinement, we use the following fixed fraction strategy: In each refinement cycle, the elements are ordered according to the size of $\eta_{T}$ and then a fixed portion of about $30 \%$ of the elements with largest $\eta_{T}$ is refined resulting in approximately a doubling of the number $N$ of cells. This process is repeated until the stopping criterion $\eta \leq T O L$ is fulfilled or $N_{\max }$ is exceeded. A more detailed discussion about mesh refinement strategies can be found, e.g., in [10].
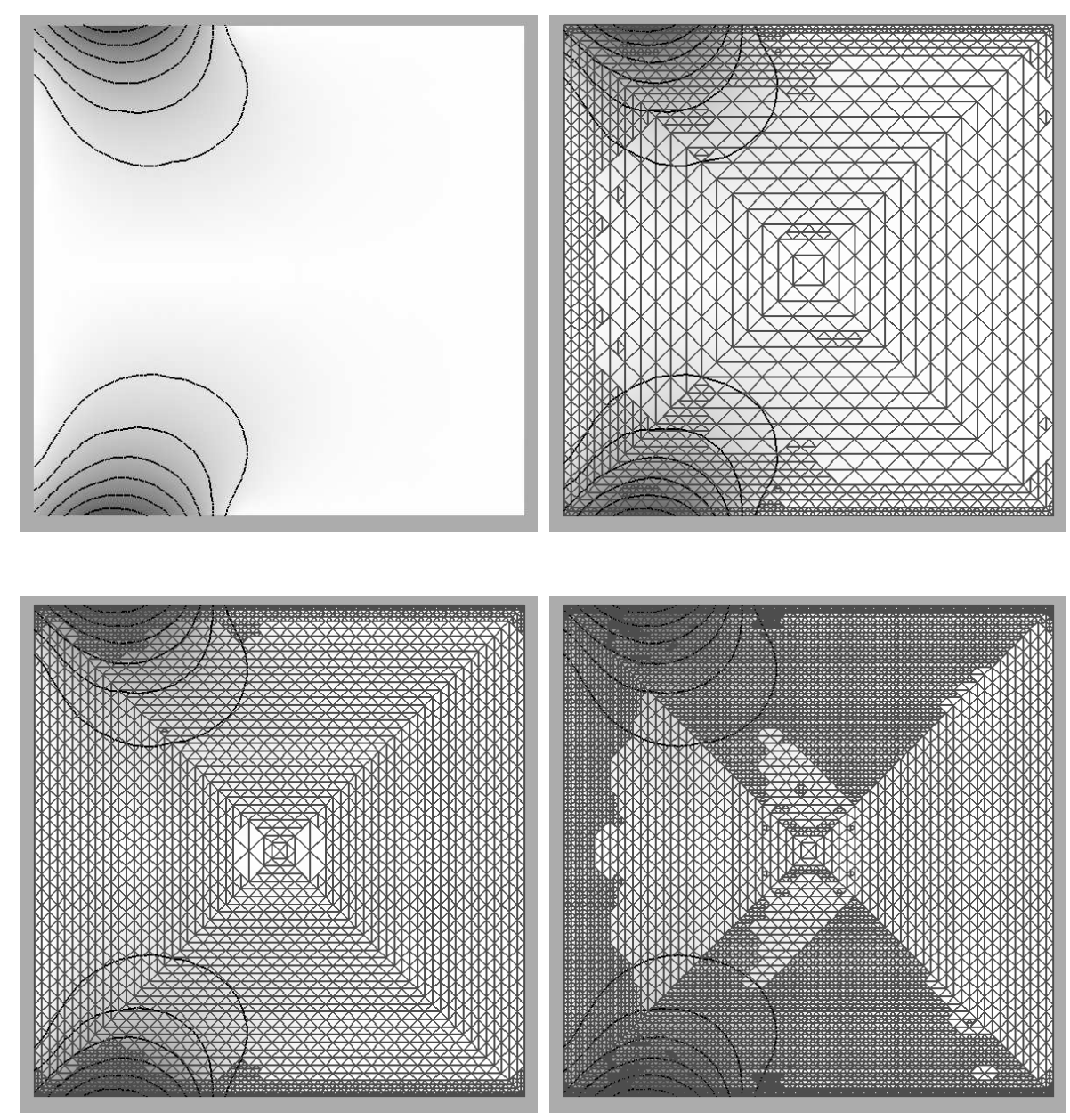

Figure 6: Sequence of locally refined grids produced by our numerical simulation, showing that especially the critical zone between contact zone and free boundary is well resolved. 


\begin{tabular}{rcc}
\hline Cells & $\eta\left(\sigma_{h}, u_{h}, f, g\right)$ & $\alpha$ \\
\hline 118 & $2.579280 \mathrm{e}+01$ & $9.112689 \mathrm{e}-01$ \\
358 & $1.512174 \mathrm{e}+01$ & $9.622250 \mathrm{e}-01$ \\
964 & $1.003769 \mathrm{e}+01$ & $8.273848 \mathrm{e}-01$ \\
2434 & $6.504695 \mathrm{e}+00$ & $9.367799 \mathrm{e}-01$ \\
5959 & $4.040411 \mathrm{e}+00$ & $1.063649 \mathrm{e}-00$ \\
\hline
\end{tabular}

Table 1: Computational results for the test example. Estimated exponents describing rates of convergence $\mathcal{O}\left(h^{\alpha}\right)$ of the proposed error estimate.

As a test example we consider the system (5) on the unit square. The external load is $f=0$. Zero boundary conditions are imposed on the vertical lines, whereas on the horizontal lines the thin obstacle is defined by $g=100 *$ $(x * x * x-1.5 * x * x+0.5 * x)$ (see Figure 7 (right)). Now based on the error estimate derived above, an adative finite element algorithm is applied to the test problem producing results on a sequence of locally refined triangulations.

Assuming $\eta\left(\sigma_{h}, u_{h}, f, g\right)$ to behave like $\mathcal{O}\left(h^{\alpha}\right)$ we determine an approximation $\alpha$ on each level, by comparing the error bounds with the estimator on the previous level. The results are listed in Table 1 . One observes $\alpha$ tending towards 1 , indicating the components of the estimate to behave like $\mathcal{O}(h)$, as one would anticipate.
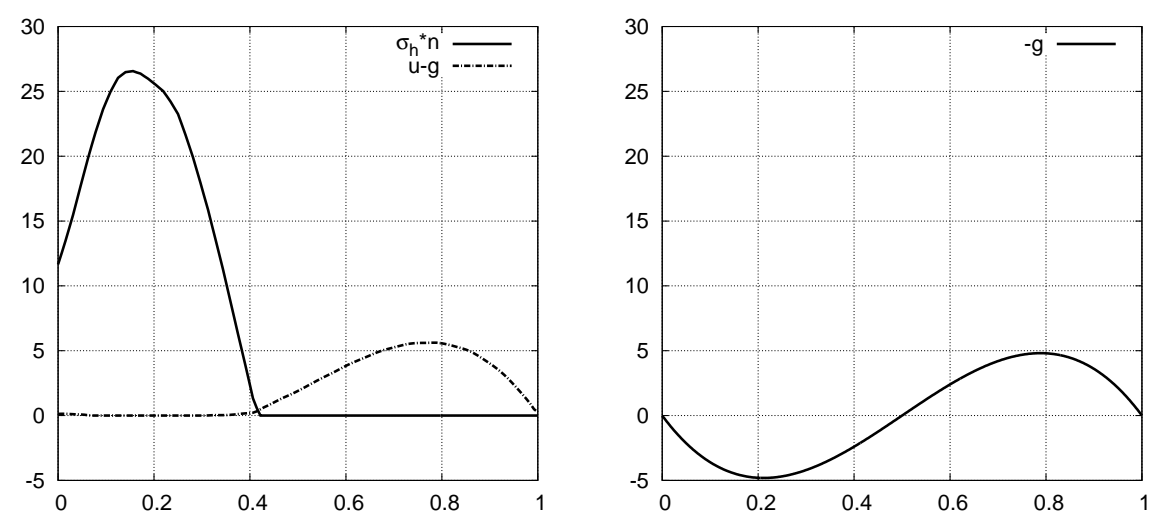

Figure 7: Snapshots along contact line: plot of $u_{L}-g$ and $\sigma_{h} \cdot n$ (left) demonstrating that the complementarity condition $(u-g) \sigma \cdot n=0$ is well approximated. Plot of the obstacle $-g$ along upper boundary (right).

Furthermore in order to illustrate that in addition the complementarity condition $(u-g) \sigma \cdot n=0$ is well approximated $u_{L}-g$ and $\sigma_{h} \cdot n$ along one contact boundary are depicted in Figure 7 (left). Eventually a sequence of adaptively refined grids, generated on the basis of the weighted estimate, is 
depicted in Figure 6. Especially the critical transition zone, where the change between contact and free boundary condition occurs, is well resolved. The background colour is given by values of $\sigma_{2}$.

\section{References}

[1] R. Becker. An Adaptive Finite Element Method for the Incompressible Navier-Stokes Equations on Time-Dependent Domains. Dissertation, Institut für Angewandte Mathematik, Universität Heidelberg, 1995.

[2] H. Blum and F.T. Suttmeier. An adaptive finite element discretisation for a simplified Signorini problem. Calcolo, 37(2):65-77, 2000.

[3] F. Brezzi, W.W. Hager, and P.A. Raviart. Error estimates for the finite element solution of variational inequalities. Numer. Math., 28:431-443, 1977.

[4] DEAL. differential equations analysis library http://www.math.uni-siegen.de/suttmeier/deal/deal.html, 1995.

[5] M. Dobrowolski and T. Staib. On finite element approximation of a second order unilateral variational inequality. Numer. Funct. Anal. and Optimiz., 13:243-247, 1992.

[6] R.S. Falk. Error estimates for the approximation of a class of variational inequalities. Math. Comp., 28:963-971, 1974.

[7] R. Glowinski. Numerical methods for nonlinear variational problems. Springer Series in Comp. Physics. Springer, 1983.

[8] N. Kikuchi and J.T. Oden. Contact Problems in Elasticity: A Study of Variational Inequalities and Finite Element Methods. Studies in Applied Mathematics 8. SIAM, 1988.

[9] J. Nitsche. $l^{\infty}$-convergence of finite element approximations. In E.Magenes I.Galligani, editor, Mathematical Aspects of Finite Element Methods, volume 606 of Lecture Notes in Math., pages 261-274. Springer-Verlag, 1977.

[10] R. Rannacher. Error control in finite element computations. Proc. NATOSummer School Error Control and Adaptivity in Scientific Computing, Antalya, Turkey, Aug. 9-12. Kluwer-Verlag, 1998.

[11] G.A. Seregin. On the regularity of weak solutions of variational problems in plasticity theory. Soviet Math. Dokl., 42(2), 1991. 
[12] P.-M. Suquet. Existence et Regularite de Solutions des Equations de la Plasticit parfaite. PhD thesis, Universite Paris Sud, 1978. These de $3^{\text {me }}$ cycle.

[13] F.-T. Suttmeier. On concepts of PDE-software: The cellwise oriented approach in DEAL. Int. Math. Forum, 2(1-4):1-20, 2007.

[14] F.T. Suttmeier. On FE-discretisations with least-squares stabilisation: A posteriori error control for the membrane-problem. EAST-WEST J. Numer. Math., 6:155-165, 1998.

[15] F.T. Suttmeier. Numerical Solution of Variational Inequalities by Adaptive Finite Elements. Vieweg+Teubner, 2008.

[16] G. Wang and X. Yang. Numerical modeling of a dual variational inequality of unilateral contact problems using the mixed finite element methood. Int. J. of numerical ananlysis and modeling, 6(1):161-176, 2009.

[17] O.C. Zienkiewicz and J.Z. Zhu. A simple error estimator and adaptive procedure for practical engineering analysis. Int. J. Numer. Methods Engrg., 24:337-357, 1987.

Received: October 21, 2013 